\title{
DECISION QUALITY IN COMPLEX PRODUCT DEVELOPMENT: REFLECTIONS ON A CASE STUDY
}

\author{
Siyam, Ghadir; \\ Salgueiro, Mariely; \\ Kennedy, John \\ bp Exploration Operating Company Limited, $\mathrm{P} \& \mathrm{O}$
}

\begin{abstract}
Conceptual design projects are increasingly known as an intense decision-making process. Much of the decision-making is comparing the degree of preference between choices. In the complex projects of the upstream business of oil and gas, good decisions are crucial for success. Decisions are typically made within a dynamic environment, wide range of uncertainty, and have to account for the asset life cycle. This paper reflects on the application of the decision quality framework with a focus on decision modelling. Using an industrial example, a systematic approach to visualise and improve decision making process is proposed. The approach applies a Dependency Structure Matrix (DSM) and Decision Quality frameworks and identified opportunities for future research.
\end{abstract}

Keywords: Decision making, Industrial design, Integrated product development

\author{
Contact: \\ Siyam, Ghadir \\ bp \\ Global Concept Development \\ United Kingdom \\ ghadir.siyam@uk.bp.com
}

Cite this article: Siyam, G., Salgueiro, M., Kennedy, J. (2021) 'Decision Quality in Complex Product Development: Reflections on A Case Study', in Proceedings of the International Conference on Engineering Design (ICED21), Gothenburg, Sweden, 16-20 August 2021. DOI:10.1017/pds.2021.86 


\section{INTRODUCTION}

Capital projects in the upstream energy industry can be complex and decision intense. The challenge in making good decisions on time emerges from the complexity of both information flow and people (including project teams and stakeholders). Moreover, when making decisions, teams need to explore multiple alternatives and their impact on the overall project, then make decisions within a wide range of uncertainty. This requires a good understanding of the dependencies between decisions and the ability to visualise the interfaces and priorities.

To improve this decision-making process, the upstream projects organisation at bp has been applying several well-established techniques within an agile working environment. This includes decision quality frameworks, decision analysis approaches and a Dependency Structure Matrix (DSM) tool. However, several gaps were identified in that some of the frameworks utilised are abstract and do not lend themselves to applied techniques for managing large and complex sets of activities. Other approaches are complicated and require external support to implement and manage the process. Moreover, the processes have been applied mainly within the internal bp team as part of the Concept Development stage and were not extended to include handover to design contractors and support the performance management of a third party by bp. Subsequently, an improved framework has evolved focusing on integrating the end user and optimising work packages as a first step, rather than optimising the full set of project decisions.

The application of a decision mapping framework has enhanced the handover clarity and team alignment during the Optimise stage, improved the overall cost and schedule planning of the project, and ensured an integrated approach to making quality decisions. Using an industrial example, we discuss in this paper our current thinking on the value of decision mapping in the Concept Development and Optimise stages and highlight opportunities for further improvement.

\section{BACKGROUND}

A decision is a situation in which a decision maker make a choice on what to do from a set of alternatives (Peterson, 2014). "Ultimately, [an organization's] value is just the sum of the decisions it makes and executes." Blenko et al, 2010. The concept development stage is an inherently decisionintense and iterative process. For example, nearly $30 \%$ of the projects tend to have either schedule slippage or substantial cost overruns (SPE, 2016). Making good decisions on time can create, or if not destroy, value for stakeholders. Hammond et al (1999) proposes a proactive decision-making approach (PrOACT) to visualise tangible and intangible aspects of decisions and translate biases and requirements into choices. The approach is based on eight elements which are divided into core (problem, objective alternatives, consequences, trade-offs) and clarity elements (uncertainty, risk tolerance, and linked decisions). The authors proposed dividing decisions to tackle complexity then systematically analysing choices. Furthermore, Spetzler et al (2016) provides a framework for making good decisions based on six requirements: appropriate frame, creative alternatives, relevant information, clear values and trade-offs, sound reasoning, and commitment. On the other hand, to improve decision quality, Sawiris et al (2015) proposes processes based on workplans that resolve uncertainties as articulated by the teams. In the Oil and Gas context, Ventroux et al. (2018) proposed a model to facilitate communication and coordination about crucial cross-boundaries decisions within and between clusters. The authors discuss improvements achieved by modelling interdependencies, identifying propagation chains and loops, and computing organizational reshuffling.

The decision quality framework is widely used in the energy sector (SPE, 2016). The aim is to support making high-quality decisions on time with optimal effort. Afterwards, effectively translate those decisions into action that create more value for stakeholder. Applying the decision quality, a need for an approach to simplify and organise decisions in a logical flow, was identified. Using a DSM approach has emerged as a feasible means to model and optimise interdependencies and, subsequently, eliminate unnecessary iteration or rework in the design process. DSM was proposed by Donald V Steward (1981) to model and optimise interdependencies and, subsequently, eliminate iteration or rework in the design process. A comprehensive list of potential DSM utilisation in planning, managing teams and decision making is discussed by Browning et al. (2006). Furthermore, Yassin and Braha (2003) discuss the linkage between the DSMs modelling of dependencies to decision making as part of a concurrent engineering practice context. By modelling these dependencies among activities, components, and people, a better understanding of dependencies can be achieved, enabling tacit 
knowledge and overcoming limitations in information processing by designers to make better decisions. Jaber et al. (2015) provides a clustering algorithm to facilitate collaborative decisionmaking process. The authors propose grouping the numerous actors shared across multiple projects according to their assigned decisions relationships.

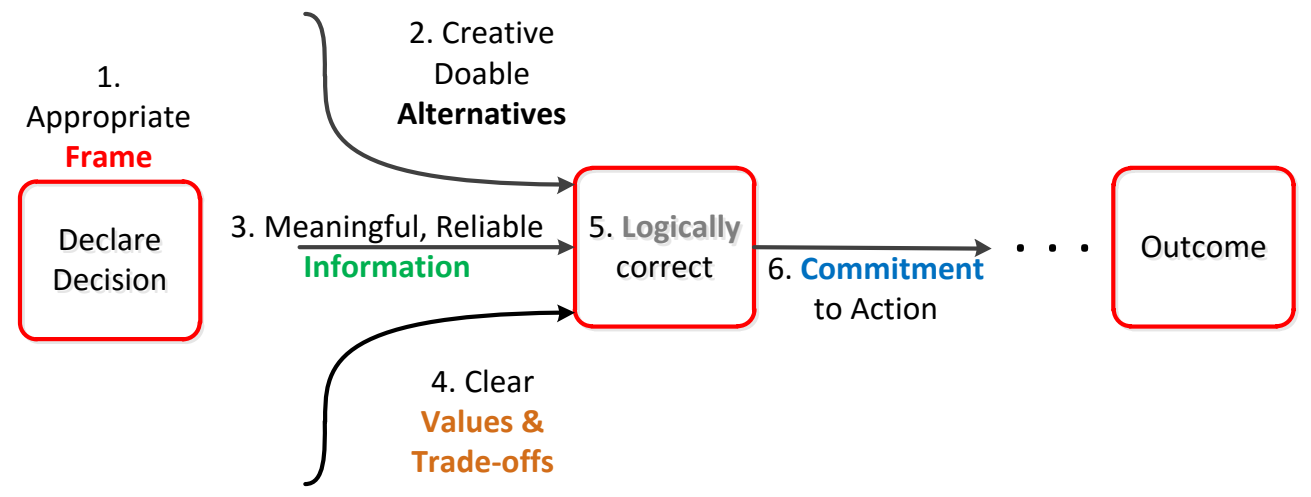

Figure 1. Decision quality process

Siyam et al (2017) and Robinson (2016) have documented the use of DSMs in planning iterative processes and decision making within the concept development. Building on these approached, further improvements are proposed to partition decisions into scopes to better visualise and map work packages against teams. Subsequent sections of this paper document and detail the application of this approach into other phases and more complex projects.

\section{MOTIVATION AND REQUIREMENTS}

Within the concept development team, much of the engineering design processes are rigorous and systematic. For example, the business framing and concept identification workshops are a standard activity in which the multidiscipline teams define scope, vision and development scenarios to ensure alignment on the project moving forward. The processes are continuously being improved and integrated within the decision quality framework. However, some gaps have been identified especially at the decision analysis, managing 3rd party process, and the team's handover from one stage to the other. Specifically, four main learnings have motivated bp to move towards decision mapping:

1. Managing interfaces and handovers between teams: Given the complexity of projects, during the handovers as a project moves from one stage to the other, a simply and rigours approach is needed to manage performance and ensure clarity on what and when decisions need to be made. This will support the team in understanding priorities to ensure efficient progress.

2. Eliminating bias: suboptimal design solution can emerge due to multiple factors including discipline bias or missing decisions dependencies. Understanding the flow of information when making decisions and documenting them into the decision papers can ensure elimination of bias and more integrated solutions.

3. Planning support: integrated teams across bp use visual boards during stand-ups to determine which activities they will work on in a daily basis and plan by weekly. The project starts with a backlog, which can include hundreds of activities. Moreover, the dependencies between these activities and rework risks are not articulated. Mapping dependencies among decisions and sequencing decisions to minimise the risk of unnecessary iterations and rework leads to efficient project delivery. This is even more critical at the current low oil prices where many projects become economically challenged; we need to focus our efforts and resources to be decision driven and value driven.

4. Engage end user: traditionally, decision analytics is done with minimum involvement of the end user after the inputs have been captured. A process to engage with more frequent input was required to ensure the process is simplified and capture requirements. Moreover, engagement was needed to ensure the output can be owned and further develop by the team as the project progresses and changes are needed. This also ensures a better utilisation of the models produced. 
Considering the learnings outlined above, a decision mapping approach to project development should recognize the importance of:

- improving the team's interface, simplifying the analysis steps, and providing team visualisation,

- consistency of results by ensuring appropriate sequencing of decisions that captures information flow as provided by the team during engagement sessions.

The appropriateness of the model would change if the characteristics of the environment change as discussed by Rahman and De Feis (2009). This model is proposed for complex decision making with high interdependencies and time pressure.

\section{DEVELOPMENT OF THE DECISION MAPPING FRAMEWORK}

Building on expertise of the application of DSMs to major capital projects in industry over the past 12 years, the framework presented below is further developed based on the evolving requirements of projects and the team. Driven by the requirements described in the previous section, the implemented approach is as follows (see figure 1):

1. Model dependencies: decisions and their associated options from the concept identification workshop are listed into a decision hierarchy. This can include up to 100 decisions, with multiple options under each. To build the DSM, the following analysis is undertaken:

- Definition of decisions - a short description of each decision and a single point of accountability are defined.

- Determine decisions dependencies - the integrated team discusses the dependencies between decision and better understand the team interactions. To facilitate this discussion, a decision DSM is used to identify dependencies in terms of (1) input/output/iterative between two decisions at a time and (2) degree of dependencies, which can be "low" if two decisions require a check-in in terms of assumptions but have no direct link, "medium" if two decisions have a "finish to finish" dependency or mid review, and "high" if a "finish to start" such that one decision requires the other to finish before starting.

- Decision Sequencing - Using the decision DSM, the decisions sequence is identified, and rework loops are highlighted. Decisions are re-sequenced to minimise iterations.

2. Classify decisions: using the DSM model developed, decisions can be classified into "work scope". On average, each work scope would include up to 10 decisions. These decisions have high interdependencies and are the accountability of a sub team/discipline within the project team. The work scopes are presented to the project team as a work structure map in order to align on expectations and ensure that the decisions are partitioned appropriately to reflect the team architecture.

3. Streamline flow: in a work session, the integrated team discusses optimisation in terms of work scopes and decisions sequencing to simplify the flow and reduce rework:

- Optimise sequence of work scopes: the dependencies between work scopes are defined by the information flow across the classified decisions. The sequence is then defined to minimise iterations/rework. Sub team's availability is used to define work scopes that can be done in parallel.

- Optimise sequence of decisions within scope: further optimisation to decisions within each work scope is undertaken by resequencing decisions to minimise iterations, identify parallel decisions and further simplify the flow.

Decisions are then reclassified if applicable and moved to different work scope in order to generate information needed for other decisions. 


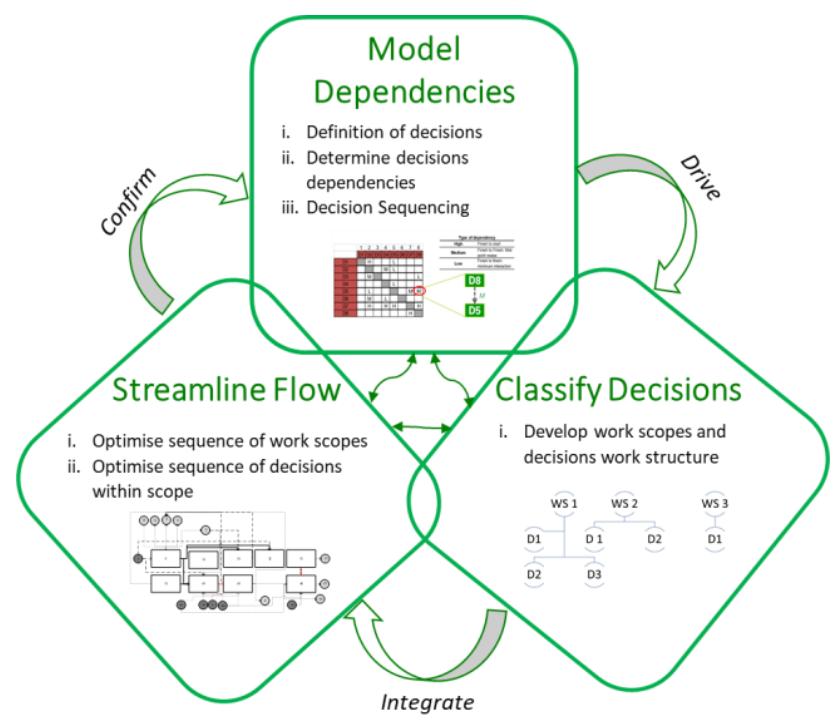

Figure 2. Decision mapping framework

\section{CASE STUDY}

The following section describes the application of the Decision Mapping Framework to a deep-water gas development. After selecting a development concept to develop a combined gas production and Liquefied Natural Gas (LNG) to market, the Project and Engineering Team needed to progress the definition of the proposed concept and optimise the development scheme to maximise value before Front-End Engineering Design (FEED). As part of the planning process for the optimisation phase, the team identified more than 60 decisions to complete prior to the detailed engineering work could start. Those decisions were classified by Tiers (1 to 3), which reflect priority and level of details and are usually associated with a specific stage in the design process. The classification is based on the impact to the project value drivers. Four decisions were identified as strategic for the development, whereas 56 decisions were identified as tactical. The large number of decisions and the perceived interdependency among those, made it difficult to the project to optimally plan work, allocate resources and develop a schedule.

As the project moved into the optimisation phase, a new team was formed and tasked to complete the engineering scope before the FEED. The plan was for the engineering work to be carried out by a 3rd party engineering contractor who would be performance managed by the bp Project Engineering Team.

Due to the complexity of the development concept, the handover of the engineering work to a new team (including 3rd party contractors), a Business Framing and Concept Identification Workshop was organised. The aim was to:

- Align the new team members and build clarity around the project scope and mission

- Review decisions by eliminating decisions or rationalising them into groups

- $\quad$ Prioritise the workflow for Pre-FEED

- Apply learnings from previous projects

The implemented approach is as follows (see figure 1):

1. Model dependencies: Following the listing of 60 decisions and their associated options by the multidisciplinary team, DSM was used to determine the interdependency and type. Figure 3 is a section of the 60x60 matrix showing dependencies type (O: Output, L: Loop, I: Input) and strength (L: Low or M: Medium or H: High). 


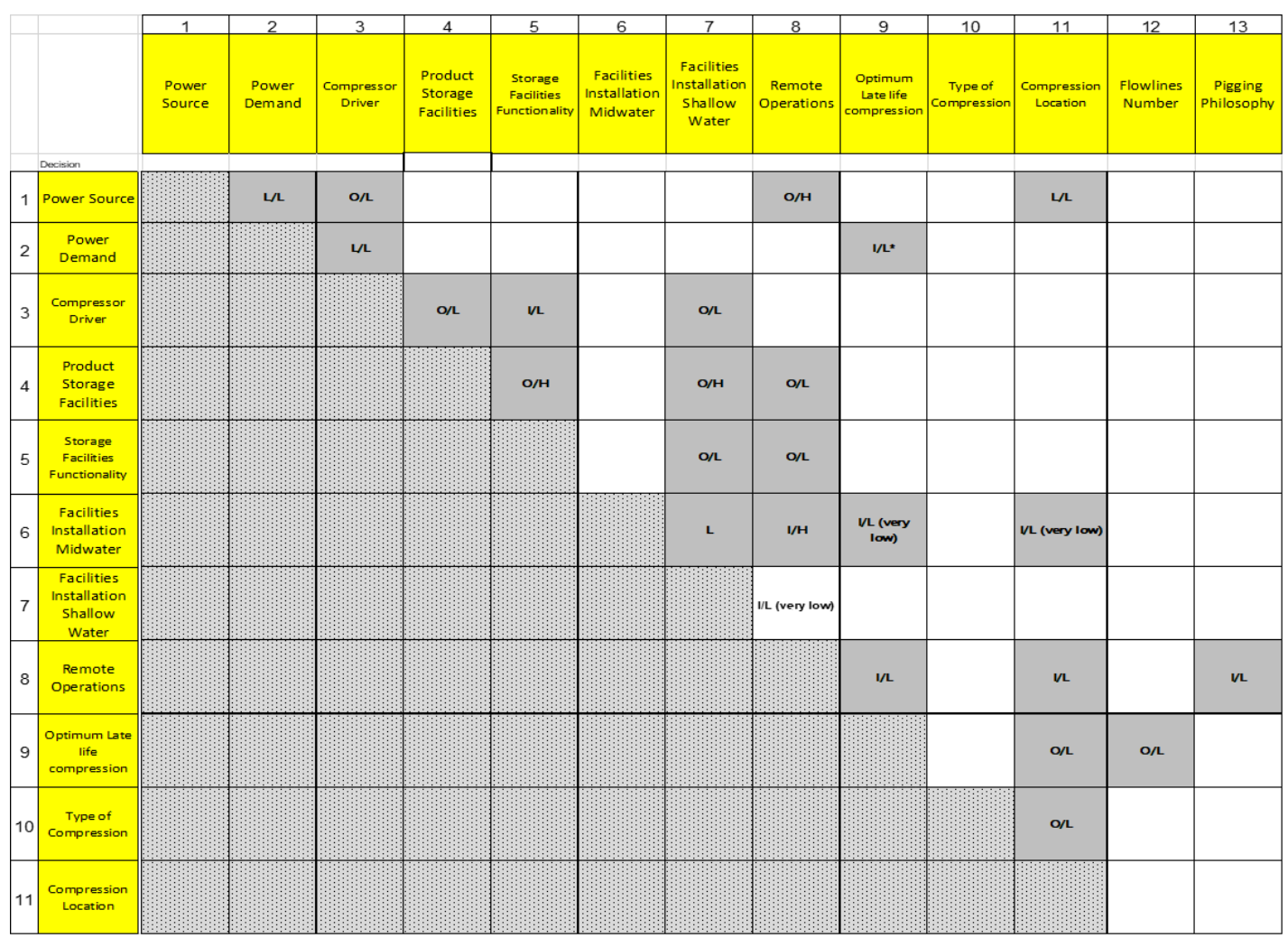

Figure 3. Short term priority decisions - interdependency matrix

After completing the Matrix for all decisions, 60 Decision Maps or Decisions Flow Diagrams were generated to demonstrate interrelationships, highlighting the complexity in generating an optimised work plan and minimise rework. The decisions were then sequenced to minimise iterations and long feedback loops. Moreover, the decisions that had no dependencies where parked and discussed by the team if they should be front loaded or delayed. Decisions that could be made in parallel were identified. The complexity of the decision flows limited the use of the output by the bp team and the 3rd Party Engineering Contractors. Therefore, a better classification was needed to simplify the process.

2. Classify decisions: at this stage, a pattern showing the groupings of decisions in which there is high dependencies was established. This grouping is referred to as "work-scopes". In total, 8 work-scopes were identified. Each work-scope had 5-13 decisions. The work-scopes and list of decisions in each was discussed in detailed with the team and modified to ensure no missing links and to reflect the team structure and availability.

3. Streamline flow Following the classification, optimisation activities were held to ensure a streamlined flow of information between scopes and within scopes' activities. To sequence the work-scopes, the external flow of decisions was used coupled with team discussions to reflect the team interactions and information availability for the front-end decisions. At a detailed level, decisions within each scope were sequenced based on the DSM model (see figure 4), where external interfaces are highlighted using the grey circles. Following the optimisation exercise, any remaining rework loops were highlighted. 


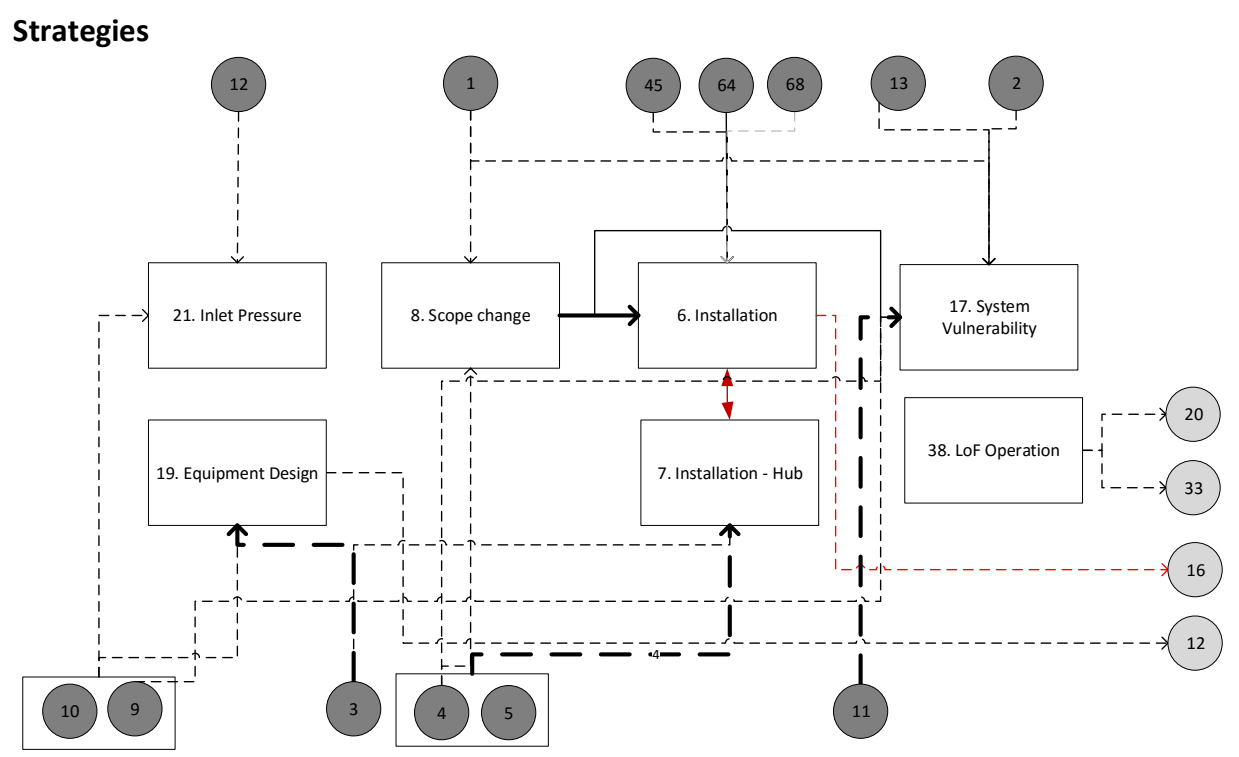

Figure 4. Optimisation of decisions within a work scope - strategies example

A final session was held by the team to confirm the classification and sequence of the overall work scopes and decisions to create a decision flow that could be understood and followed by all engineering disciplines involved. The team also shared the results with the third party and investigated ways to integrate it into their standard business tools and workflows. The updated streamline flow is shown in figure 5 .

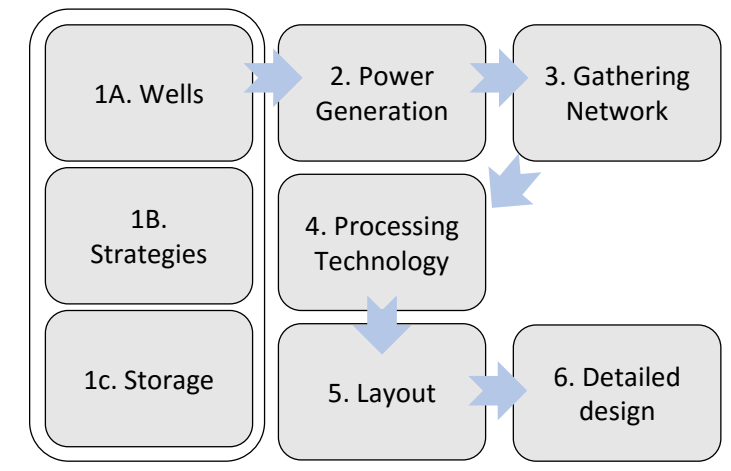

Figure 5. Streamline flow of work scopes for a deep-water development

In summary, the simplified decision Map as well as the Interdependency Matrix, were used to;

- Plan and prioritise work-scopes, beyond the simplified original short-term/long term classification.

- Focus on completion of key decisions that unlock resolution of subsequent, more tactical decisions

- Aid decision framing by describing the interdependencies that need to be considered when scoping the work required to close a decision.

- Identify stakeholders in the decision-making process: giving a clear understanding of interdependencies between decisions allowed interfaces between disciplines, contractors, central function, and partners to be identified.

- Aid communication with 3rd party contractors and other stakeholders.

- Aid agile visualization tools to track progress and prioritise work

- Embed Decision Quality in the decision-making process by allowing decisions to be made in logical order.

Opportunities achieved by applying the framework are as following:

- The framework proposed in this paper enables prioritisation of critical decisions that can unlock the greatest value or represent the grates risk to the project during the early stages. 
- The simplicity and transparency of the process by which the decision map was generated minimised stakeholder biases towards a particular outcome. Interdependency between decisions and identification of critical decisions defined the order and priority to which decisions should be executed.

- Handover between delivery teams of a complex project with a high degree of interdependency between decisions was simplified and more effective by applying the proposed framework.

- This framework complements agile techniques by providing a technique to optimise work and schedule whilst visualising progress.

- Finally, this framework further supports and enhances the benefits or decision-based planning by driving the project team to focus, prioritise and allocate the right amount of resources to the critical decisions that will bring most value to the project during the Optimise stage.

\section{DISCUSSION}

This section reflects upon the development and utilisation of the decision mapping framework. Limitations and future potential research are outlined below.

Firstly, in section 3, four key requirements emerged from the previous work and identified by the team during the planning session. The framework proposed in this paper meets these requirements as follows:

1. Managing interfaces and handovers between teams: by modelling the relationships between activities, product components, and people, a better understanding of dependencies can be obtained, enabling tacit knowledge of these development elements to become explicit. The framework highlights interfaces between different functions and enables a better handover through a collaborative development of the model.

2. Eliminating bias: "Humans often make less than optional decisions from a rational viewpoint" (Phillips-Wren and Power, 2019). Systems need to be underpinned by statistics, mathematical, and computer-science research to help decision maker think rationally. As the model proposed here was built and owned by the multidisciplinary team, changes and decisions were methodical following the framework logic and not entirely dependent on experience.

3. Planning support: the framework developed by the team was used as part of the visual board and linked into the backlog. The prioritised decisions based on functions were subsequently translated into set of activities in the backlog during daily team stand-ups.

4. Engage end user: team engagement started in the business framing workshop followed by the concept identification session. The team, as end users of the framework, were engaged to define the decisions, describe options, define information flow, and optimise the sequence. This engagement gave a sense of ownership and a better understanding to optimise the model as requirements or more information become available.

Secondly, any model developed is only as good as its inputs and assumptions. In the engineering design space, with limited information, the outcome of decisions made can only be determined in later stages. The confidence level in assumptions can be improved with further data and tests. Moreover, the model was built such that it provides flexibility to the team to change sequence and options as the project progresses, allowing for assumption quality to be continuously reviewed. In general, to ensure quality of assumptions, the discussion included at least one functional tag to provide the needed input and was reviewed by engineering managers and subject matter experts. Moreover, lessons learned from previous projects was captured as appropriate and incorporated into the model by the team.

Thirdly, time was invested to plan and develop the model. The build duration was 2 weeks, resulting from a request by the engineering lead to develop a model for handover to the project team. The two weeks involved the decision identification session and multiple 1-hour review discussions with the cross functional team to engage and further develop ideas. This duration enabled the team members to align on the scope of work and engineering studies requested. Therefore, the time invested in planning at the front end ensured a common foundation and ownership by the team moving forward.

Fourthly, there is value in applying the method in an industrial context. DSM has been generally applied in multiple industrial contexts and is commonly used by the industry. Applying the framework proposed in the early phases of complex product development can potentially support teams in better framing projects. Moreover, it supports the Agile way of working that is being heavily adopted by industries in the engineering design space. 
Next, the team investigated opportunities for continuous improvement and in doing so came across several limitations:

- Classification of dependency strength in practice was challenging: for each two decisions, the team was asked about the dependencies type (O: output, L: loop, I: input) and strength, which can be "low" indicating a check-in in terms of assumptions but have no direct link, "medium" indicating a "finish to finish" and "high" for a "finish to start". In practice, this was proven challenging to articulate as much of the information flow between decisions can be highly interdependent. Therefore, during the DSM exercise, indirect dependencies that can be captured through other decisions were omitted. Moreover, after building the model, the team reviewed each work scope as a story board to ensure dependencies reflect the expected logic based on prior experience.

- Translating decisions into activities: The framework is limited to prioritising decisions. However, in projects a workplan is required based on activities to manage progress. Deriving activities from decisions to ensure a decision focused plan can be challenging due to the added complexity in the level of details, activity sequencing, and ownership. A practical model to manage activities within decisions can be timely and cost effective to streamline the process of delivering the concepts.

- Supporting technology to help with the analysis: although multiple software exists to build DSMs and build a 3D decision models, it remains complex and requires to be maintained by a practitioner rather than the end user. A simple and flexible technology to help the team managing these frameworks is needed.

- Measuring impact on performance: measuring the benefits of these models and time invested in modelling them is challenging. Future studies can support in articulating the value proposition to enable further adaptation of these frameworks.

- Measuring quality of assumptions: as discussed earlier, this is one of the challenges in the engineering design phase. The framework proposed does not incorporate a confidence level or management of change.

- Differentiating between dependencies and readiness: in this framework, DSM discussion captures mainly the flow of information. However, some of the studies are time dependent, for example results of drilling wells and analysing dynamic data. The dependencies in the framework does not capture the readiness of the decision in terms of the availability of all input data required to make a decision.

Finally, reflecting on the limitations discussed above, two main future research questions are proposed that would enable better adoption of these frameworks in general in industries. Firstly, exploring decision-based project plans that are practical and manageable. Secondly, reflecting on readiness level of decisions as part of the DSM dependency discussions.

\section{CONCLUSIONS}

The complexity of Oil and Gas projects has increased over recent years, with new developments posing technical challenges that require decisions to be made in a timely manner and in the correct order to maximise value for investors. New projects need to compete for portfolio resources and require optimisation to be the "best they can be" before investors commit to execution. The framework presented in this paper seeks to provide a simplified approach to optimising decision analysis and mapping. The framework integrates Decision Quality framework with DSM to support team performance management. Feedback from applying the framework in a complex project suggests that the approach is promising for industrial application as it unlocks value, simplifies processes, improves handovers, and streamlines workflows.

\section{ACKNOWLEDGMENTS}

This paper would not have been possible without the project team Paul Brennan (Mechanical Engineer) and Hemita Morar (Process Engineer) for their exceptional contribution to producing the matrix and maps, and for Fintan O'neill (Engineering Manager), Mark Tatum (Engineering Manager), and Nathan Barret (Engineering Director) for their support in implementing the process. This work builds on the years of previous work in applying DSMs at bp supported by Bob Robinson, and the decision quality committee supported by Chris Travis (Appraisal General Manager). 


\section{REFERENCES}

Blenko, M.W., Mankins, M.C., and Rogers, P. (2015), "The Decision-Driven Organization", Harvard business Review, section 4.

Browning, T.R., Fricke, E., and Negele, H. (2006), "Key Concepts in Modeling Product Development Processes", Systems Engineering, Vol. 9 No. 2, pp. 104-128.

Carl Spetzler, Hannah Winter, Jennifer Meyer (2016), "Decision Quality: Value Creation from Better Business Decisions" John Wiley \& Sons, Business \& Economics

Eppinger, S.D. (2001), "Innovation at the Speed of Information", Harvard Business Review, Vol. 79 No. 1, pp. 149-158.

Hammond, John S., Ralph L. Keeney, and Howard Raiffa(1999), "Smart Choices: A Practical Guide to Making Better Decisions" New York: Broadway Books

Jaber, H., Marle, F., Jankovic, M., (2015), "Improving Collaborative Decision Making in New Product Development Projects Using Clustering Algorithms," IEEE Transactions on Engineering, Management. Volume: 62, Issue: 4, Page(s): 475 - 483

Peterson, M. (2014), "Decision Theory: AN Introduction", Springer, Berlin

Phillips-Wren, G. and Power, D., (2019), "Cognitive bias, decision styles, and risk attitudes in decision making and DSS", Journal of Decision Systems

Rahman, N. and De Feis, G., (2009), "Strategic Decision-Making: Models and Methods in the Face of Complexity and Time Pressure", Journal of General Management

Robinson, B. (2015), "Decision Road mapping: Decision Making at Pace", International Dependency and Structure Modelling Conference (DSM '15), Fort Worth, Texas.

1. R. Sawiris, C. S. Howes, J. A. Rodriguez, W. L. Foley(2015), "Uncertainty and Risk Management Plans are Critical for Team Alignment and Better Decision Quality", SPE-174932-MS, SPE Annual Technical Conference and Exhibition, 28-30 September, Houston, Texas, USA

Siyam, Ghadir; Robinson, Robert Wilson; and Kilpinen, Malia (2017), "Modelling Decisions in Complex Products", 21 st International Conference on Engineering Design, ICED 17, Vancouver, Canada

Steward, Donald V (1981), "The design structure system: A method for managing the design of complex systems," IEEE Transactions on Engineering Management, vol. EM-28, no. 3, pp. 71-74, Aug. 1981. https://dx.doi.org/10.1109/TEM.1981.6448589

Ventroux, J., Franck M., and Vidal, L. (2018), " Organizational reshuffling to facilitate coordinated decisions in complex projects", Concurrent Engineering, Volume: 26 issue: 3, page(s): 299-309

Yassin, A. and Braha, D. (2003), "Complex Concurrent Engineering and the Design Structure Matrix Method", Concurrent Engineering, Vol. 11 No. 3, pp. 165-176. 